\title{
Análisis del sistema de gestión ambiental de un operador portuario del terminal de contenedores del Puerto del Callao
}

\author{
Assessment of the environmental management system of a container terminal port operator of the \\ Port of Callao
}

\author{
Sergio Pacsi Valdivia ${ }^{1 *} ;$ Diego Manya Gutiérrez ${ }^{2}$ \\ *Autor de correspondencia
}

\section{Resumen}

La presente investigación tiene como objetivo, evaluar la implementación del sistema de gestión ambiental (SGA) de un Operador Portuario del Terminal de Contenedores del Puerto del Callao-Perú, con énfasis en la correcta identificación de los aspectos e impactos ambientales y en la vinculación de los componentes básicos del SGA para su control y seguimiento. Para ello, se identificó y evaluó los aspectos e impactos ambientales del operador portuario, utilizando la metodología de la Matriz de Leopold. Los aspectos e impactos ambientales determinados fueron comparados con la matriz de evaluación de aspectos e impactos ambientales del SGA del operador portuario. Los resultados muestran que la organización en estudio no ha identificado completamente los impactos ambientales de sus operaciones en la implementación de su SGA y no cuenta con mecanismos de control para sus impactos ambientales identificados. El análisis de los componentes del SGA vinculados a los aspectos e impactos ambientales de la organización, indica que existe ausencia de los elementos de seguimiento adecuados para los posibles aspectos e impactos ambientales de la organización.

Palabras Clave: Sistema de gestión ambiental; ISO 14001; Aspectos ambientales; Impactos ambientales; Gestión ambiental; Desempeño ambiental.

\begin{abstract}
This research aims to evaluate the implementation of the Environmental Management System (EMS) of a Container Terminal Port Operator of the Port of Callao-Peru; with emphasis on the correct identification of environmental aspects and impacts, and on the linkage of the EMS basic components for its control and monitoring. For this purpose, the environmental aspects and impacts of the port operator were identified and evaluated, using the Leopold Matrix methodology. The specific environmental aspects and impacts were compared with the matrix assessment of environmental aspects and impacts of the port operator's EMS. The results show that the organization under study has not completely identified the environmental impacts of its operations in the EMS implementation and does not have control mechanisms for its identified environmental impacts. The analysis of the components of the EMS related to the environmental aspects and impacts of the organization indicates that there is no adequate monitoring elements for possible environmental aspects and impacts of the organization.
\end{abstract}

Keywords: Environmental management systems; ISO 14001; Environmental aspects; Environmental impacts; Environmental management; Environmental performance.

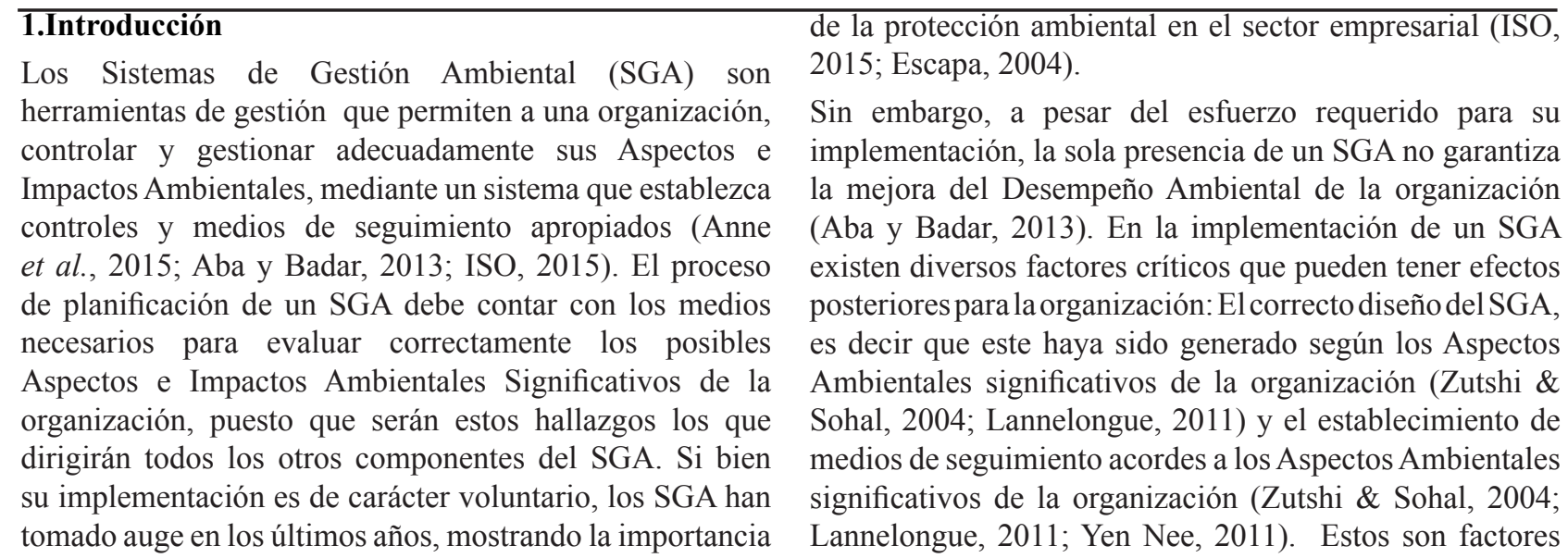

${ }^{1}$ Facultad de Ciencias de la Universidad Nacional Agraria La Molina, Apartado postal 12-056, La Molina, Lima (Perú). Email: spv@lamolina.edu.pe ${ }^{2}$ Especialistas del Servicio Nacional de Certificación Ambiental para las Inversiones Sostenibles - SENACE, Lima, Perú 
críticos en el éxito de la implementación de un SGA y se encuentran estrechamente vinculados al futuro desempeño ambiental de una organización.

Sin embargo, a pesar de esta importancia con respecto a este componente del diseño de un SGA, la norma ISO 14001 no establece como requisito el uso de una metodología en particular sino que deja esta evaluación a criterio de la organización (ISO, 2015; Aba y Badar, 2013). Debido a esta salvedad, cada organización evalúa sus Aspectos e Impactos Ambientales según sus propios métodos y consideraciones, causando que en muchos casos las metodologías usadas no cuenten con una base teórica fundamentada y estén sujetas al criterio del evaluador.

De forma opuesta a esta heterogeneidad, en la evaluación de Impactos Ambientales, fuera del marco del SGA existen métodos definidos y con solidez científica usados para los procesos de Evaluaciones de Impacto Ambiental (EIA), procesos que también buscan la evaluación de los efectos sobre el medio ambiente de las actividades de un proyecto u organización (Pereira et al., 2014). Los Estudios de Impacto Ambiental y Sistemas de Gestión Ambiental son herramientas de protección ambiental complementarias, si bien sus orígenes y las condiciones de su ejecución pueden variar, sus objetivos fundamentales son similares (Palframan, 2010). A pesar de ello, ambas herramientas no cuentan en la práctica con una vinculación estrecha a pesar de la gran similitud entre los elementos de ejecución de un EIA y de planificación de un SGA, lo que sugiere que el primero puede ser usado como parte de la información base para el establecimiento y diseño adecuado de un SGA (Sánchez \& Hacking, 2002), principalmente en el proceso de Identificación de Aspectos e Impactos Ambientales Significativo del SGA.

En la presente investigación se analizó el diseño e implementación del SGA de un Operador Portuario del Terminal Marítimo de Contenedores del Puerto del Callao - Perú, con énfasis en los elementos vinculados a la Identificación de Aspectos e Impactos Ambientales, mediante un proceso comparativo entre la identificación de Impactos Ambientales en el marco de un EIA y la evaluación de aspectos e impactos ambientales según lo establecido por el SGA de la organización. Finalmente se verificó la implementación de los medios de seguimiento establecidos para los aspectos ambientales identificados y las carencias que puedan detectarse de aquellos impactos que no fueron considerados.

\section{Materiales y métodos}

\section{Evaluación del diseño del sistema de gestión ambiental del operador portuario}

A fin de evaluar el diseño del SGA del Operador Portuario del Terminal de Contenedores, se estableció un marco metodológico que permitiera analizar la correcta identificación de los Aspectos Ambientales Significativos y sus medios de control asociados. Este análisis se dio mediante las siguientes dos etapas:
Etapa 1: Identificación de Aspectos e Impactos Significativos de la organización:

Usando los resultados del Sistema de Gestión Ambiental de la organización y la información especializada de investigaciones realizadas sobre la evaluación del SGA realizados en otros países (NIOT, 2003; Nawrocka y Parker, 2009; Escapa, 2004; EPA, 2004; Lannelongue, 2011), se procedió a identificar los potenciales Impactos Ambientales causados por la organización en estudio. Este análisis se realizó mediante el uso del método de la Matriz de Leopold (Leopold et al., 1971; Neacsu y DaneciPatrau, 2014). El método se basa en un cuadro donde se comparan un total de 100 actividades (Columnas) con un total de 88 posibles efectos ambientales (Filas). Sin embargo considerando que muchas de estas actividades no son aplicables a todas las organizaciones, esta matriz fue modificada de forma que se consideraran las actividades aplicables a un terminal marítimo de contenedores, de forma similar se consideró necesario agregar aquellas actividades que la organización realizaba pero que no se encontraran listadas en los actividades estándar de la matriz de Leopold, esto con el fin de hacer la evaluación lo más exhaustiva posible. Las actividades evaluadas (columnas) y los posibles efectos ambientales (filas), se muestran en las Tablas 1 y 2 respectivamente.

Tabla 1. Actividades a ser evaluadas en la Matriz de Leopold

\begin{tabular}{|c|c|}
\hline Actividad & Sub-actividad \\
\hline $\begin{array}{l}\text { A. Modificación del } \\
\text { régimen }\end{array}$ & $\begin{array}{l}\text { Introducción de flora o fauna } \\
\text { exótica } \\
\text { Ruidos y vibraciones }\end{array}$ \\
\hline B. Alteración del terreno & Dragado de cuerpos \\
\hline C. Cambios en el tráfico & $\begin{array}{l}\text { Camiones } \\
\text { Barcos }\end{array}$ \\
\hline $\begin{array}{l}\text { D. Tratamiento y vertido } \\
\text { residuos }\end{array}$ & $\begin{array}{l}\text { Vertidos en el mar } \\
\text { Vertidos de efluentes líquidos } \\
\text { Explosiones }\end{array}$ \\
\hline E. Accidentes & $\begin{array}{l}\text { Incendios } \\
\text { Escapes y fugas de materiales } \\
\text { peligrosos } \\
\text { Operaciones en puerta de } \\
\text { ingreso/salida vehicular } \\
\text { Operaciones marítimas } \\
\text { (buque/muelle) } \\
\text { Operaciones en patio de } \\
\text { contenedores } \\
\text { Zona de aforo / } \\
\text { almacenamiento temporal }\end{array}$ \\
\hline
\end{tabular}

F. Otras actividades Mantenimiento ingeniería de grúas Mantenimiento - ingeniería de equipos móviles Mantenimiento de infraestructura Almacén general Zona de abastecimiento de combustible Edificio administrativo

Fuente: Adaptado de Leopold (1971) 
Los potenciales impactos ambientales fueron identificados según su posible causa, ya sea Directa (D) en el caso de las actividades propias ejecutadas por la organización, o Indirecta (I) según se den por acciones vinculadas a terceras partes pero a raíz de la presencia de la organización. Finalmente, luego de ejecutado el análisis se procedió a comparar los resultados del estudio con la Matriz de Evaluación de Aspectos e Impactos de la organización a fin de verificar que impactos ambientales no habían sido identificados durante la implementación del SGA de la organización.

Tabla 2. Efectos Ambientales a ser evaluados en la Matriz de Leopold

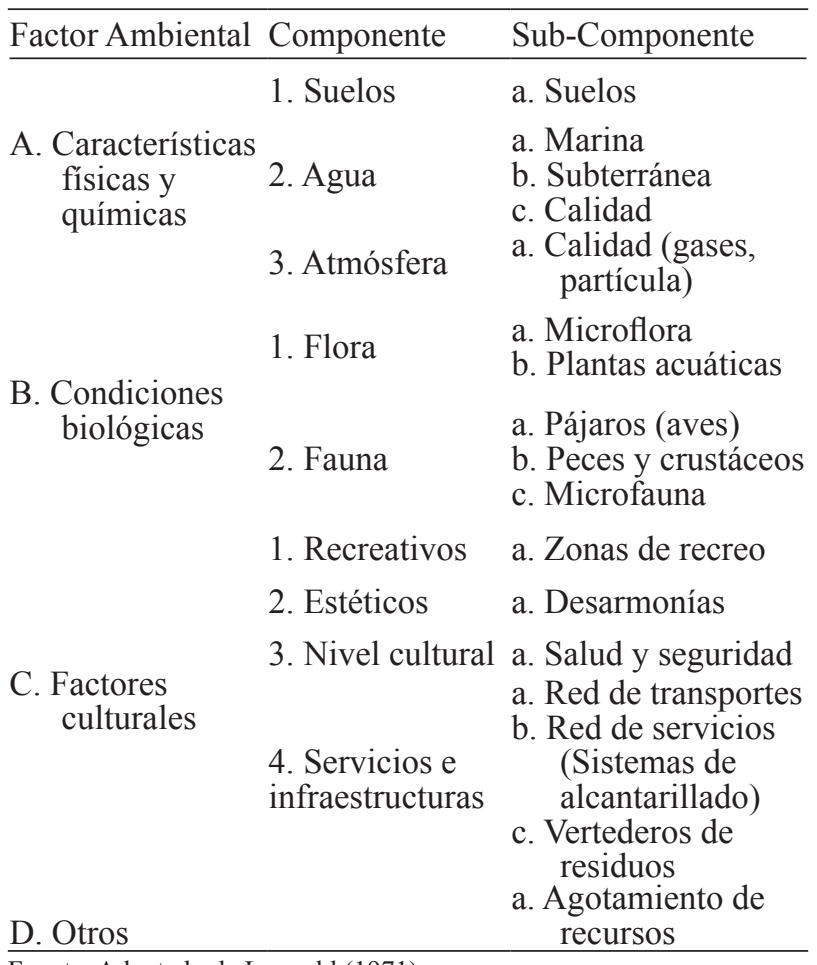

Fuente: Adaptado de Leopold (1971).

Etapa 2: Evaluación de los Componentes del Sistema de Gestión Ambiental

Luego de identificar los Aspectos e Impactos Ambientales de la organización, se verificaron en la documentación del SGA, la presencia de elementos de control para estos componentes y medios de seguimiento asociados a los Aspectos Ambientales Significativos. Este análisis, se realizó mediante la revisión de toda la documentación del SGA, en particular la vinculada al establecimiento de programas, objetivos y metas.

\section{Resultados y discusión}

Identificación de Aspectos e Impactos Significativos de la organización:

Los resultados de la identificación de los aspectos e impactos ambientales significativos de la organización, mediante la aplicación del método de matriz de Leopold se detallan en la Tabla 3 . Los resultados de la matriz 150 de Leopold fueron comparados con los resultados del proceso interno de identificación de aspectos e impactos ambientales significativos de la organización. Este análisis permitió determinar si la organización identificó adecuadamente sus propios impactos ambientales y por tanto si el SGA está correctamente dimensionado. La Tabla 4 muestra aquellos impactos ambientales que no fueron identificados por la organización y que por consiguiente no cuentan con las medidas de control establecidas en el marco del SGA. El análisis realizado permitió identificar que la organización no ha evaluado todos los potenciales impactos de sus actividades, y que si bien ha ejecutado una gran tarea en la ejecución de este análisis, este aún requiere de una actualización y revisión. Es importante notar que uno de los impactos ambientales no evaluados está vinculado al manejo de aguas de lastre de las naves, el cual si bien es una actividad de los buques, esta indirectamente relacionada a la presencia y actividades de la instalación portuaria. La problemática de las aguas de lastre es un aspecto ambiental muy severo a nivel global y que puede tener efectos negativos en los ecosistemas donde el agua de lastre no es bien manejada (OMI, 2014). Si bien no es el objetivo de la investigación evaluar la metodología usada por la organización para la identificación de sus aspectos ambientales, es importante destacar que la consideración de solo cinco tipos de impactos como parte de la evaluación de aspectos ambientales, provocó que otros elementos vinculados al ámbito de los impactos sociales, no hayan sido considerados y evaluados en el marco de su metodología, pudiendo esto también ser una causa de las deficiencias halladas.

\section{Evaluación de los Componentes del Sistema de Gestión Ambiental}

Luego de haber ejecutado el análisis comparativo de los Impactos Ambientales de la organización; se procedió a verificar si los impactos considerados originalmente por esta, cuentan con componentes dentro del SGA que permitan su control y seguimiento. En la Tabla 5, se muestra la relación entre los Aspectos Ambientales de la organización y los componentes del SGA que buscan su control y seguimiento. El análisis de los componentes del SGA vinculados a los aspectos e impactos ambientales de la organización muestra que a pesar de haber identificado distintos aspectos e impactos ambientales significativos, la organización no cuenta con los medios adecuados para ejecutar el seguimiento de su control. Esto es particularmente notorio con el Aspecto Ambiental "Potenciales Derrames" donde si bien se cuenta con un elemento de control operativo como es el "Plan de Emergencia de la Instalación Portuaria", no se cuenta con objetivos establecidos en el marco del cumplimiento de este plan, ni mucho menos con indicadores que permitan medir el desempeño de la organización en el control de los "Potenciales Derrames" ni en los impactos ambientales que estos podrían generar, como contaminación del suelo, contaminación del agua, o los posibles efectos sobre las poblaciones cercanas. 
Tabla 3. Identificación de Potenciales Impactos Ambientales de la organización

\begin{tabular}{|c|c|c|c|c|c|c|c|}
\hline & & & \multicolumn{2}{|c|}{$\begin{array}{l}\text { Modificación del } \\
\text { régimen }\end{array}$} & \multirow{2}{*}{$\begin{array}{c}\begin{array}{c}\text { Alteración } \\
\text { del terreno }\end{array} \\
\text { Dragado de } \\
\text { cuerpos }\end{array}$} & \multicolumn{2}{|c|}{$\begin{array}{c}\text { Cambios en el } \\
\text { tráfico }\end{array}$} \\
\hline & & & $\begin{array}{l}\text { Introducción } \\
\text { de flora o } \\
\text { fauna exótica }\end{array}$ & $\begin{array}{c}\text { Ruidos y } \\
\text { vibraciones }\end{array}$ & & Camiones & Barcos \\
\hline \multirow{5}{*}{$\begin{array}{l}\text { Características } \\
\text { fisicas y } \\
\text { químicas }\end{array}$} & 1. Suelos & a. Suelos & - & - & - & $\mathrm{I} / \mathrm{D}$ & - \\
\hline & & a. Marina & I & - & $\mathrm{D}$ & - & I \\
\hline & 2. Agua & b. Subterránea & & - & & - & - \\
\hline & & a. Calidad & I & - & $\mathrm{D}$ & - & I \\
\hline & 3. Atmósfera & $\begin{array}{l}\text { a. Calidad (gases, } \\
\text { partícula) }\end{array}$ & - & - & - & $\mathrm{D}$ & I \\
\hline \multirow{3}{*}{$\begin{array}{l}\text { Condiciones } \\
\text { biológicas }\end{array}$} & 1. Flora & $\begin{array}{l}\text { a. Microflora } \\
\text { b. Plantas acuáticas }\end{array}$ & I & - & $\mathrm{D}$ & - & I \\
\hline & & a. Pájaros (aves) & & $\mathrm{D}$ & - & $\mathrm{D}$ & I \\
\hline & 2. Fauna & $\begin{array}{l}\text { b. Peces y crustáceos } \\
\text { c. Microfauna }\end{array}$ & I & - & $\mathrm{D}$ & - & - \\
\hline \multirow{6}{*}{$\begin{array}{l}\text { Factores } \\
\text { culturales }\end{array}$} & 1. Recreativos & a. Zonas de recreo & - & $\mathrm{D}$ & - & - & - \\
\hline & $\begin{array}{l}\text { 2. Estéticos y } \\
\text { de interés } \\
\text { humano }\end{array}$ & a. Desarmonías & - & - & - & - & - \\
\hline & 3. Nivel cultural & a. Salud y seguridad & - & $\mathrm{D}$ & - & $\mathrm{D}$ & - \\
\hline & & a. Red de transportes & - & - & - & I & - \\
\hline & $\begin{array}{l}\text { 4. Serviciose } \\
\text { infraestructuras }\end{array}$ & $\begin{array}{l}\text { b. Red de servicios } \\
\quad \text { (sistemas de } \\
\text { alcantarillado público) }\end{array}$ & - & - & - & - & - \\
\hline & & c. Vertederos de residuos & - & - & $\mathrm{D}$ & - & - \\
\hline Otros & & $\begin{array}{l}\text { a. Agotamiento de } \\
\text { recursos }\end{array}$ & - & - & $\mathrm{D}$ & $\mathrm{D}$ & - \\
\hline
\end{tabular}

\begin{tabular}{|c|c|c|c|c|c|c|c|}
\hline & & & \multicolumn{2}{|c|}{$\begin{array}{c}\text { Tratamiento y vertido de } \\
\text { residuos }\end{array}$} & \multicolumn{3}{|c|}{ Accidentes } \\
\hline & & & $\begin{array}{l}\text { Vertidos en el } \\
\text { mar }\end{array}$ & $\begin{array}{l}\text { Vertidos de } \\
\text { efluentes } \\
\text { líquidos }\end{array}$ & Explosiones & Incendios & $\begin{array}{l}\text { Escapes } \\
\text { y fugas }\end{array}$ \\
\hline \multirow{5}{*}{$\begin{array}{l}\text { Características } \\
\text { físicas y } \\
\text { químicas }\end{array}$} & 1. Suelos & a. Suelos & - & - & - & - & - \\
\hline & & b. Marina & I & - & - & - & $\mathrm{D}$ \\
\hline & 2. Agua & a. Subterránea & - & - & - & - & $\mathrm{D}$ \\
\hline & & b. Calidad & I & - & - & - & $\mathrm{D}$ \\
\hline & 3. Atmósfera & $\begin{array}{l}\text { a. Calidad (gases, } \\
\text { partícula) }\end{array}$ & - & - & $\mathrm{D}$ & $\mathrm{D}$ & $\mathrm{D}$ \\
\hline \multirow{3}{*}{$\begin{array}{l}\text { Condiciones } \\
\text { biológicas }\end{array}$} & 1. Flora & $\begin{array}{l}\text { a. Microflora } \\
\text { b. Plantas acuáticas }\end{array}$ & I & - & - & - & - \\
\hline & & a. Pájaros (aves) & - & - & $\mathrm{D}$ & $\mathrm{D}$ & $\mathrm{D}$ \\
\hline & 2. Fauna & $\begin{array}{l}\text { b. Peces y crustáceos } \\
\text { c. Microfauna }\end{array}$ & I & - & - & - & $\mathrm{D}$ \\
\hline \multirow{6}{*}{$\begin{array}{l}\text { Factores } \\
\text { culturales }\end{array}$} & 1. Recreativos & a. Zonas de recreo & - & - & $\mathrm{D}$ & $\mathrm{D}$ & $\mathrm{D}$ \\
\hline & $\begin{array}{l}\text { 2. Estéticos y de } \\
\text { interés humano }\end{array}$ & a. Desarmonías & - & - & $\mathrm{D}$ & $\mathrm{D}$ & $\mathrm{D}$ \\
\hline & 3. Nivel cultural & a. Salud y seguridad & - & - & $\mathrm{D}$ & $\mathrm{D}$ & $\mathrm{D}$ \\
\hline & & a. Red de transportes & - & - & - & - & - \\
\hline & $\begin{array}{l}\text { 4. Servicios e } \\
\text { infraestructuras }\end{array}$ & $\begin{array}{l}\text { b. Red de servicios } \\
\text { (sistemas de } \\
\text { alcantarillado público) }\end{array}$ & - & $\mathrm{D}$ & - & - & $\mathrm{D}$ \\
\hline & & c. Vertederos de residuos & $\mathrm{I} / \mathrm{D}$ & $\mathrm{D}$ & - & - & $\mathrm{D}$ \\
\hline Otros & & $\begin{array}{l}\text { a. Agotamiento de } \\
\text { recursos }\end{array}$ & - & - & - & - & - \\
\hline
\end{tabular}

Fuente: Elaboración Propia, en base a Neacsu y Daneci-Patrau (2014)

Tipo de impacto: $\mathrm{I}=$ indirecto, $\mathrm{D}=$ directo 
Continuación de Tabla 3

\begin{tabular}{|c|c|c|c|c|c|c|}
\hline & & & & Otras ac & ctividades & \\
\hline & & & $\begin{array}{c}\text { Operaciones } \\
\text { en puerta de } \\
\text { vehicular }\end{array}$ & $\begin{array}{c}\text { Operaciones } \\
\text { maritimas } \\
\text { (buque/muelle) }\end{array}$ & $\begin{array}{l}\text { Operaciones } \\
\text { en patio de } \\
\text { contenedores }\end{array}$ & $\begin{array}{l}\text { Zona de aforo / } \\
\text { almacenamiento } \\
\text { temporal }\end{array}$ \\
\hline & 1. Suelos & a. Suelos & & & $\mathrm{I} / \mathrm{D}$ & \\
\hline & & a. Marina & & $\mathrm{I} / \mathrm{D}$ & & \\
\hline Caracteristicas & 2. Agua & b. Subterránea & & $\mathrm{I} / \mathrm{D}$ & & \\
\hline $\begin{array}{l}\text { fisicas y } \\
\text { auímicas }\end{array}$ & & c. Calidad & & & & \\
\hline & 3. Atmósfera & $\begin{array}{l}\text { a. Calidad (gases, } \\
\text { partícula) }\end{array}$ & $\mathrm{I} / \mathrm{D}$ & $\mathrm{I}$ & $\mathrm{I} / \mathrm{D}$ & $\mathrm{D}$ \\
\hline & 1. Flora & $\begin{array}{l}\text { a. Microflora } \\
\text { a. Plantas acuáticas }\end{array}$ & & I & & \\
\hline $\begin{array}{l}\text { Condiciones } \\
\text { biológicas }\end{array}$ & & a. Pájaros (aves) & $\mathrm{I} / \mathrm{D}$ & $\mathrm{I} / \mathrm{D}$ & $\mathrm{I} / \mathrm{D}$ & $\mathrm{D}$ \\
\hline & 2. Fauna & $\begin{array}{l}\text { b. Peces y crustáceos } \\
\text { c. Microfauna }\end{array}$ & & I & & \\
\hline & 1. Recreativos & a. Zonas de recreo & & & & \\
\hline & $\begin{array}{l}\text { 2. Estéticos y de } \\
\text { interés humano }\end{array}$ & a. Desarmonías & & & & \\
\hline & 3. Nivel cultural & a. Salud y seguridad & $\mathrm{I} / \mathrm{D}$ & & $\mathrm{I} / \mathrm{D}$ & \\
\hline Factores & & a. Red de transportes & I & & & \\
\hline culturales & $\begin{array}{l}\text { 4. Servicios e } \\
\text { infraestructuras }\end{array}$ & $\begin{array}{l}\text { b. Red de servicios } \\
\text { (sistemas de } \\
\text { alcantarillado } \\
\text { público) }\end{array}$ & & & & $\mathrm{D}$ \\
\hline & & $\begin{array}{l}\text { c. Vertederos de } \\
\text { residuos }\end{array}$ & $\mathrm{D}$ & $\mathrm{D}$ & & $\mathrm{D}$ \\
\hline Otros & & $\begin{array}{l}\text { a. Agotamiento de } \\
\text { recursos }\end{array}$ & $\mathrm{D}$ & $\mathrm{D}$ & $\mathrm{D}$ & $\mathrm{D}$ \\
\hline & & & & Otras ac & ctividades & \\
\hline & & & $\begin{array}{r}\text { Mantenimic } \\
\text { ingeniería de } \\
\text { móvile. }\end{array}$ & $\begin{array}{l}\text { ento - Mant } \\
\text { equipos ingenie } \\
S\end{array}$ & $\begin{array}{l}\text { tenimiento } \\
\text { ería de grúas }\end{array}$ & $\begin{array}{c}\text { Mantenimiento de } \\
\text { infraestructura }\end{array}$ \\
\hline & 1. Suelos & a. Suelos & $\mathrm{D}$ & & $\mathrm{D}$ & $\mathrm{D}$ \\
\hline & & a. Marina & & & & $\mathrm{D}$ \\
\hline Características & 2. Agua & b. Subterránea & $\mathrm{D}$ & & $\mathrm{D}$ & $\mathrm{D}$ \\
\hline fisicas y & & c. Calidad & & & & $\mathrm{D}$ \\
\hline & 3. Atmósfera & $\begin{array}{l}\text { a. Calidad (gases, } \\
\text { partícula) }\end{array}$ & $\mathrm{D}$ & & $\mathrm{D}$ & $\mathrm{D}$ \\
\hline & 1. Flora & $\begin{array}{l}\text { a. Microflora } \\
\text { b. Plantas acuáticas }\end{array}$ & & & & \\
\hline $\begin{array}{l}\text { Conalclones } \\
\text { biológicas }\end{array}$ & & a. Pájaros (aves) & $\mathrm{D}$ & & $\mathrm{D}$ & $\mathrm{D}$ \\
\hline & 2. Fauna & b. Peces y crustáceos & & & & \\
\hline & 1. Recreativos & a. Zonas de recreo & & & & \\
\hline & $\begin{array}{l}\text { 2. Estéticos y de } \\
\text { interés humano }\end{array}$ & a. Desarmonías & & & & \\
\hline & 3. Nivel cultural & a. Salud y seguridad & & & & \\
\hline Factores & & a. Red de transportes & & & & \\
\hline culturales & $\begin{array}{l}\text { 5. Servicios e } \\
\text { infraestructuras }\end{array}$ & $\begin{array}{l}\text { b. Red de servicios } \\
\text { (sistemas de } \\
\text { alcantarillado } \\
\text { público) }\end{array}$ & $\mathrm{D}$ & & $\mathrm{D}$ & $\mathrm{D}$ \\
\hline & & $\begin{array}{l}\text { c. Vertederos de } \\
\text { residuos }\end{array}$ & $\mathrm{D}$ & & $\mathrm{D}$ & $\mathrm{D}$ \\
\hline Otros & & $\begin{array}{l}\text { a. Agotamiento de } \\
\text { recursos }\end{array}$ & D & & D & D \\
\hline
\end{tabular}




\begin{tabular}{|c|c|c|c|c|c|}
\hline & & & & K. Otras activid & ades \\
\hline & & & $\begin{array}{l}\text { Almacén } \\
\text { general }\end{array}$ & $\begin{array}{c}\text { Zona de } \\
\text { abastecimiento } \\
\text { de combustible }\end{array}$ & $\begin{array}{c}\text { Edificio } \\
\text { administrativo }\end{array}$ \\
\hline & 1. Suelos & a. Suelos & $\mathrm{D}$ & $\mathrm{D}$ & \\
\hline & & a. Marina & & & \\
\hline $\begin{array}{l}\text { Caracterlstlcas fislcas } \\
\text { y químicas }\end{array}$ & 2. Agua & b. Subterránea & $\mathrm{D}$ & $\mathrm{D}$ & $\mathrm{D}$ \\
\hline & & c. Calidad & & & \\
\hline & 3. Atmósfera & a. Calidad (gases, partícula) & $\mathrm{D}$ & $\mathrm{D}$ & $\mathrm{D}$ \\
\hline & 1. Flora & $\begin{array}{l}\text { a. Microflora } \\
\text { b. Plantas acuáticas }\end{array}$ & & & \\
\hline Condiciones & & a. Pájaros (aves) & $\mathrm{D}$ & $\mathrm{D}$ & \\
\hline & 2. Fauna & b. Peces y crustáceos & & & \\
\hline & & c. Microfauna & & & \\
\hline & 1. Recreativos & a. Zonas de recreo & & & \\
\hline & $\begin{array}{l}\text { 2. Estéticos y de } \\
\text { interés humano }\end{array}$ & a. Desarmonías & & & \\
\hline Factores culturales & 3. Nivel cultural & a. Salud y seguridad & & $\mathrm{D}$ & \\
\hline & & a. Red de transportes & & & \\
\hline & $\begin{array}{l}\text { 1. Servicios e } \\
\text { infraestructuras }\end{array}$ & $\begin{array}{l}\text { c. Red de servicios (sistemas } \\
\text { de alcantarillado público) }\end{array}$ & $\mathrm{D}$ & $\mathrm{D}$ & $\mathrm{D}$ \\
\hline & & b. Vertederos de residuos & $\mathrm{D}$ & $\mathrm{D}$ & $\mathrm{D}$ \\
\hline Otros & & A. Agotamiento de recursos & $\mathrm{D}$ & $\mathrm{D}$ & $\mathrm{D}$ \\
\hline
\end{tabular}

Tabla 4. Impactos Ambientales No Considerados por la organización

\begin{tabular}{|c|c|c|c|c|}
\hline $\begin{array}{c}\text { Componente } \\
\text { Ambiental }\end{array}$ & $\begin{array}{c}\text { Sub- } \\
\text { Componente }\end{array}$ & Elemento & Detalle & Análisis \\
\hline \multirow{3}{*}{$\begin{array}{l}\text { Características } \\
\text { físicas y químicas }\end{array}$} & \multirow{3}{*}{ 1. Agua } & \multirow{2}{*}{ a. Marina } & $\begin{array}{l}\text { Impacto por evacuación } \\
\text { de Agua de Lastre con } \\
\text { especies foráneas. }\end{array}$ & $\begin{array}{l}\text { La organización no ha evaluado los } \\
\text { impactos de la descarga de agua de } \\
\text { lastre de las naves amarradas a su } \\
\text { muelle }\end{array}$ \\
\hline & & & $\begin{array}{l}\text { Impacto por actividades } \\
\text { de mantenimiento del área } \\
\text { marina de la instalación } \\
\text { (Dragado) }\end{array}$ & $\begin{array}{l}\text { La organización no ha evaluado } \\
\text { los impactos de las actividades de } \\
\text { mantenimiento por dragado. }\end{array}$ \\
\hline & & b. Subterránea & $\begin{array}{l}\text { Impacto por Derrames en el } \\
\text { Suelo que puedan llegar a } \\
\text { contaminar la napa freática } \\
\text { Intrusión salina por exceso } \\
\text { de consumo de agua } \\
\text { subterránea. }\end{array}$ & $\begin{array}{l}\text { La organización no ha evaluado } \\
\text { los impactos de la descarga } \\
\text { de contaminantes en las aguas } \\
\text { subterráneas, asimismo no ha } \\
\text { evaluado los impactos de una posible } \\
\text { sobreexplotación del acuífero que } \\
\text { usan. }\end{array}$ \\
\hline
\end{tabular}


Continuación de Tabla 4

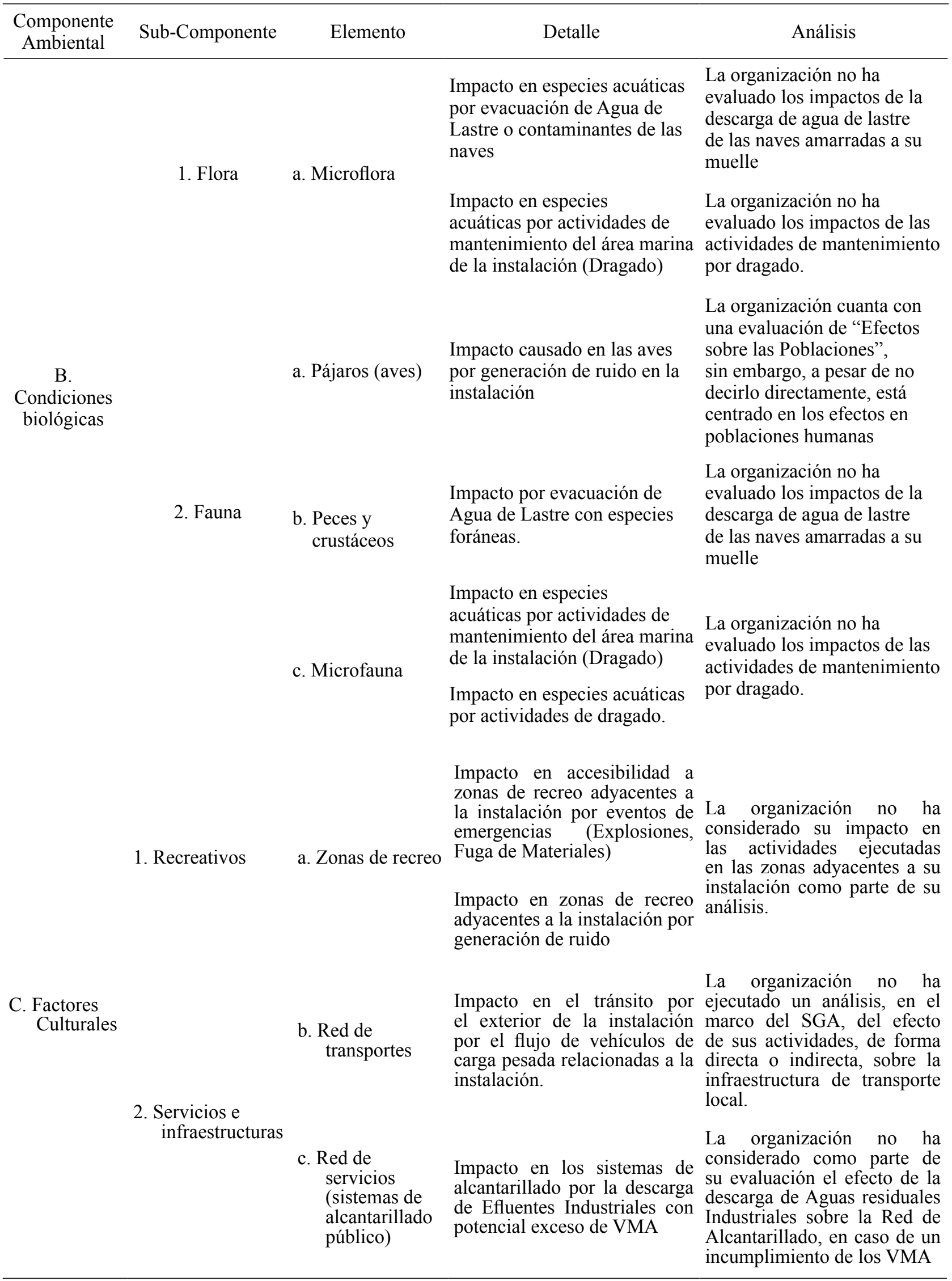


Por otro lado, se han identificado otros aspectos e impactos ambientales que tienen además de los medios de control operativo, mecanismos de seguimiento periódico establecido como indicadores. Este hecho permite a la organización conocer de forma periódica su desempeño ambiental con respecto a este aspecto o impacto ambiental, sin embargo, esto se dará únicamente en el caso que los indicadores se encuentren adecuadamente definidos y correctamente calculados con respecto a aquello que pretenderían medir. impactos ambientales significativos de una organización, lo cual afectaría su desempeño ambiental.

\section{Literatura citada}

Aba, E. K. \& Badar, M. A. 2013. A review of the impact of ISO 9000 and ISO 14000 certifications. Journal of Technology Studies, 39(1): 42-50. Disponible en: https://search.proquest.com/docview/1465330576?acc ountid $=43847$.

Tabla 5. Aspectos Ambientales y Componentes del SGA asociados

\begin{tabular}{|c|c|c|c|c|}
\hline $\begin{array}{l}\text { Aspecto } \\
\text { Ambiental }\end{array}$ & Impacto Ambiental & $\begin{array}{l}\text { Programas de Gestión } \\
\text { Ambiental }\end{array}$ & $\begin{array}{l}\text { Objetivos de } \\
\text { Desempeño } \\
\text { Ambiental }\end{array}$ & $\begin{array}{l}\text { Indicadores de Desempeño } \\
\text { Ambiental }\end{array}$ \\
\hline $\begin{array}{l}\text { Generación de } \\
\text { Residuos }\end{array}$ & $\begin{array}{l}\text { Presión sobre } \\
\text { rellenos }\end{array}$ & $\begin{array}{l}\text { Programa de Manejo de } \\
\text { Residuos Sólidos }\end{array}$ & $\mathrm{Si}$ & $\begin{array}{l}\text { Total de Residuos Comunes } \\
\text { Generados. } \\
\text { Porcentaje del Aceites Reciclados. }\end{array}$ \\
\hline $\begin{array}{l}\text { Consumo de } \\
\text { Recursos }\end{array}$ & $\begin{array}{l}\text { Agotamiento de } \\
\text { recursos }\end{array}$ & $\begin{array}{l}\text { Programa de Medición y } \\
\text { Disminución de Recursos y } \\
\text { Emisiones de GEI }\end{array}$ & $\mathrm{Si}$ & $\begin{array}{l}\text { Emisiones Totales de GEI. } \\
\text { Cantidad de Papel Consumido. } \\
\text { Consumo de Agua. }\end{array}$ \\
\hline $\begin{array}{l}\text { Generación de } \\
\text { Ruido }\end{array}$ & $\begin{array}{l}\text { Contaminación } \\
\text { Acústica }\end{array}$ & $\begin{array}{l}\text { Programa de Monitoreo y } \\
\text { Control del ruido ambiental. }\end{array}$ & No & $\begin{array}{l}\text { Cantidad de Incumplimientos } \\
\text { Injustificados de los ECA de } \\
\text { Ruido. }\end{array}$ \\
\hline $\begin{array}{l}\text { Emisión } \\
\text { de Gases } \\
\text { y Material } \\
\text { Particulado }\end{array}$ & $\begin{array}{l}\text { Contaminación del } \\
\text { Aire }\end{array}$ & $\begin{array}{l}\text { Programa de Monitoreo y } \\
\text { Control de la Calidad del } \\
\text { aire. }\end{array}$ & $\mathrm{Si}$ & $\begin{array}{l}\text { Cantidad de Incumplimientos } \\
\text { Injustificados de los ECA de Aire. } \\
\text { Porcentaje Total de Unidades } \\
\text { Externas con Inspección } \\
\text { Ambiental. }\end{array}$ \\
\hline \multirow{3}{*}{$\begin{array}{l}\text { Potenciales } \\
\text { Derrames }\end{array}$} & $\begin{array}{l}\text { Contaminación del } \\
\text { suelo }\end{array}$ & \multirow{3}{*}{$\begin{array}{l}\text { Plan de Emergencia de la } \\
\text { Instalación portuaria. }\end{array}$} & No & - \\
\hline & $\begin{array}{l}\text { Contaminación del } \\
\text { Agua }\end{array}$ & & No & - \\
\hline & $\begin{array}{l}\text { Efectos Sobre } \\
\text { Poblaciones } \\
\text { Cercanas }\end{array}$ & & No & - \\
\hline
\end{tabular}

\section{Conclusiones}

La evaluación realizada al diseño del SGA mediante la comparación de los impactos ambientales identificados en la implementación del SGA de la organización y el análisis realizado con el método de la Matriz de Leopold, indica que la organización en estudio no ha identificado completamente los aspectos e impactos ambientales de sus operaciones en la implementación de su SGA y no cuenta con mecanismos de control para todos sus impactos ambientales identificados. El análisis de los componentes del SGA vinculados a los aspectos e impactos ambientales de la organización, indica que existe ausencia de los elementos de seguimiento y control adecuados para los aspectos e impactos ambientales de la organización. En consecuencia, el inadecuado diseño de la implementación del SGA y el uso de un método muy general para la identificación y evaluación de los aspectos ambientales, puede generar errores en la etapa de evaluación de los
Anne, O.; Burskyte, V.; Stasiskiene, Z. \& Balciunas, A. 2015. The influence of the environmental management system on the environmental impact of seaport companies during an economic crisis: Lithuanian case study. Environmental Science and Pollution Research International, 22(2): 1072-1084. doi:http://dx.doi. org/10.1007/s11356-014-3410-x.

Environmental Protection Agency (EPA), (2004), An Environmental Management System (EMS) Primer for Ports: Advancing Port Sustainability, $50 \mathrm{p}$.

Escapa, C., 2004, Motivations And Barriers Of Implementing An Ems In Spanish Organizations, Tesis Ms. C. Norwick, Uk, University Of East Anglia, 112 p.

ISO. 2015. ISO 14001:2015. Environmental management systems - Requirements with guidance for use. Third edition 2015-09-15. 35 p. Disponible en: https:/www. 
iso.org/obp/ui\#iso:std:iso:14001:ed-3:v1:en

Lannelongue, G. 2011, Esfuerzo y Eficacia en los Sistemas de Gestión Medioambiental de Empresas Certificadas ISO 14001, Tesis Ph. D., Salamanca, España, Universidad De Salamanca, 374 P.

Leopold, Lb; Clarke, Fe; Hanshaw, Bb; Balsley, Jr. 1971. A Procedure for Evaluating Environmental Impact. 16 p.

NIOT. 2003. National Institute Of Ocean Technology. EIA Guidelines for Ports and Harbours, $227 \mathrm{p}$.

Nawrocka, D.; Parker, T. 2009. Finding the connection: environmental management systems and environmental performance, Journal of Cleaner Production 17: 601607.

Neacsu, G. \& Daneci-Patrau, D. 2014. Leopold Matrix and Risk Matrix Used In the Management of Environmental Impact. Economics, Management and Financial Markets, 9(4), 262-274. Retrieved from https://search.proquest.com/docview/1650863255?acc ountid $=43847$.

Organización Marítima Internacional (OMI). 2014, Manejo de Aguas de Lastre, Consultado el 23 de Feb 2014, Disponible en: http://www.imo.org/OurWork/ Environment/BallastWaterManagement/Pages/ Default.aspx.

Palframan, L. (2010),.“The integration of environmental impact assessment and environmental management systems: experiences from the UK.", $6 \mathrm{p}$.

Pereira, D. H.; Ferreira, L. F.; de Souza, M. M. \& Ferreira, D. D. M. 2014. Matriz De Aspectos E Impactos Ambientais Como Ferramenta De Evidenciação De Contingências Ambientais. Revista De Gestão Ambiental e Sustentabilidade, 3(2), 74-91. Disponible en: https://search.proquest.com/docview/1699271096? accountid $=43847$.

Sánchez, L. \& Hacking, T. 2002. An approach to linking environmental impact assessment and environmental management systems. Impact Assessment and Project Appraisal, 20(1): 25-38.

Yen Nee, G. 2011. Determining Factors for ISO14001 EMS Implementation among SMEs in Malaysia: A Resource Based View, World Academy of Science, Engineering and Technology, 59:1294-1299.

Zutshi, A. y Sohal, S. 2004. Adoption and maintenance of environmental management systems: critical success factors, Management of environmental quality, 15(4):399-419. 